This item is the archived peer-reviewed author-version of:

\title{
Review : chromatography as an inspiration for microreactors
}

\section{Reference:}

Hereijgers Jonas, Breugelmans Tom, De Malsche Wim.- Review : chromatography as an inspiration for microreactors Journal of chemical technology and biotechnology / Society of Chemical Industry [London] - ISSN 0268-2575 - (2015), p. 1-33 Full text (Publishers DOI): http://dx.doi.org/doi:10.1002/jctb.4772

To cite this reference: http://hdl.handle.net/10067/1265720151162165141 


\title{
Review: Chromatography as an
}

\section{inspiration for microreactors}

\author{
Jonas Hereijgers ${ }^{\mathrm{a}, \mathrm{b}}$, Tom Breugelmans ${ }^{\mathrm{b}}$ and Wim De Malsche ${ }^{\mathrm{a}^{*}}$ \\ ${ }^{a}$ Department of Chemical Engineering, Vrije Universiteit Brussel, Pleinlaan 2, 1050 Brussel, Belgium \\ ${ }^{b}$ Faculty of Applied Engineering, Research group Advanced Reactor Technology, University of \\ Antwerp, Salesianenlaan 90, 2660 Hoboken, Belgium
}

Email: jhereijg@vub.ac.be, tom.breugelmans@uantwerpen.be,wdemalsc@vub.ac.be

*Corresponding author: tel. (+) 32/ 2.629.33.18, fax (+) 32/ 2.629.32.48, e-mail:

wdemalsc@vub.ac.be

This article has been accepted for publication and undergone full peer review but has not been through the copyediting, typesetting, pagination and proofreading process, which may lead to differences between this version and the Version of Record. Please cite this article as doi: $10.1002 / \mathrm{jctb} .4772$

This article is protected by copyright. All rights reserved. 


\begin{abstract}
In the shift from batch to continuous operations, microreactors, at least in academic research, have become an important segment. One of the next steps in process intensification is believed to be the reduction of axial dispersion, which will not only yield higher efficiencies but also allows to integrate the reactor unit with downstream processing. Chromatographic (micro)devices are highly interesting in this context as they successfully combine a narrow residence time distribution with a high separation factor. In the present review the low dispersion in microreactors is discussed, with an emphasis on throughput, packing and flow distribution units. Subsequently, the relevance and progress during the last decade of combined reaction and separations in chromatographic and membrane based systems is treated.
\end{abstract}

\title{
1. Introduction
}

Since roughly 2 decades process intensification (PI) has dramatically gained importance in the field of chemical engineering. One of the main focusses of PI was the shift from batch to continuous processes for a number of reasons, such as improved process control. With the widespread availability of micro-precision technologies and their great conceptual promise with respect to heat and mass transfer and high-throughput operation, microreactors have received a large amount of attention both by the academic community as the industry. As a result, processes were successfully transformed from a batch to a continuous operation (1), often resulting in a substantial increase of the efficiency, e.g. due to usage of shortcuts in the reaction mechanism by new routes that were inaccessible in batch mode (e.g. flash chemistry (2)). A next important step forward in PI can be made by reducing axial dispersion, which in a second step can also allow to integrate downstream processing into the reactor unit, of which reactive distillation in traditional macroscale systems is a typical example. Combining reaction and separation can lead to unprecedented efficiency gains, but near plug flow behavior will always be critical when pushing the performance to its limits as a large dispersion will otherwise diminish the obtained gain. High performance liquid chromatography is highly interesting in this respect as it very successfully combines these two features. For optimal chromatographic separations, dispersion should be as low as possible on the one hand, while a sufficiently large separation factor should be achieved on the other hand.

This article is protected by copyright. All rights reserved. 
In the following sections, we will first (section 2) discuss the relevance of a lack of dispersion in microreactors with an emphasis on throughput, packing and flow distribution units. Subsequently, the relevance and progress during the last decade of combined reactions and separations in chromatographic and membrane based systems will be treated (sections 3).

\section{Plug flow}

Through miniaturization high mass transfer rates are obtained and because of short lateral diffusion times the axial dispersion in a single microchannel is small. To increase the throughput multiple channels can be placed in parallel (numbering-up) or widened (scaling-up), preferably without inducing a new dispersion source. When the interfacing channels vary considerably or when the fluid is maldistributed the advantage of the plug-flow behavior of the microreactor deteriorates. As a result, each molecule will spend a different time inside the microreactor, resulting in a substantial residence time distribution (RTD), quantified by Eqs. 1 \& 2(3):

$$
\begin{gathered}
E(t / \tau) \cong 0.5 \sqrt{\frac{B o}{\pi t / \tau}} e^{-\frac{(1-t / \tau)^{2} B o}{4 t / \tau}} \\
B o=\frac{u L}{D_{a x}}
\end{gathered}
$$

with $\mathrm{E}$ the exit age distribution (or residence time distribution), $\mathrm{t}$ the residence time, $\tau$ the mean residence time, Bo the Bodenstein number, $u$ the velocity, $L$ the length of the reactor and $D_{a x}$ the axial dispersion coefficient. The Bodenstein number is the ratio of convective transport to dispersion(4) and thus at infinitely large Bo (in practice above $B o=100)(3)$ an ideal plug flow reactor is obtained and at Bo equal to zero the reactor is perfectly mixed. To calculate the axial dispersion coefficient different models can be used, depending on the operating conditions. At very low flow rates the model uniquely based on diffusion (Eq. 3) can be used:

$$
D_{a x}=D_{m}
$$

This article is protected by copyright. All rights reserved. 
with $D_{m}$ the molecular diffusion coefficient. In general the dispersion model is more appropriate and the axial dispersion coefficient is then described by the Taylor-Aris correlation(5,6) (Eq. 4). The dispersion model however does not take disorder and non-laminar flow profiles into account. Whereas the former requirements is generally met when the packing and coating is ordered (7), the latter assumption is often not due to advection effects that already occur at Re numbers of 1 and above $(8,9)$.

$$
D_{a x}=D_{m}+\frac{u^{2} d_{h}^{2}}{\kappa D_{m}}
$$

with $d_{h}$ the hydraulic diameter and $\kappa$ a geometric factor (192 for circular tubes and 119 for rectangular tubes). The residence time distribution (RTD) in a reactor is a characteristic feature for chemical reactions that take place inside the reactor (4). Moreover, it affects the conversion and for instance gains in importance with consecutive reactions $(A \rightarrow B \rightarrow C)$. At a broad $R T D$ a part of $B$ remains excessively long in the reactor and will further convert to $\mathrm{C}$. Hence, a narrow RTD is beneficial to maximize the throughput, as with decreasing RTD the conversion increases (Eq. 5). This can be seen when the conversion is calculated for an ideal plug flow reactor (PFR) and a tubular laminar flow reactor (TLFR). For a PFR the RTD is given by the dirac delta function (Eq. 6), resulting in Eq. 7 for the conversion for a first order reaction. For a TLFR the RTD is given by Eq. 8 and the conversion can be calculated by Hilder's approximation (Eq. 9) (10) for a first order reaction. From Fig. 1 it is clear that an increase in RTD results in a lower conversion.

$$
\begin{aligned}
& \frac{d \bar{X}}{d t}=X(t) E(t) \\
& \text { PFR } \quad E(t)=\delta(t-\tau) \quad(6) \quad \bar{X}=1-e^{-k \tau} \\
& \text { TLFR } \quad E(t)=\left\{\begin{array}{cc}
0 & t<\frac{\tau}{2} \\
\frac{\tau^{2}}{3 t^{3}} & t \geq \frac{\tau}{2}
\end{array} \quad \text { (8) } \quad \bar{X}=\frac{(4+k \tau) e^{0.5 k \tau}+k \tau-4}{(4+k \tau) e^{0.5 k \tau}+k \tau}\right.
\end{aligned}
$$

With $\mathrm{X}$ the conversion, $\delta$ the dirac delta function, $\mathrm{t}$ the residence time, $\tau$ the space time and $\mathrm{k}$ the reaction rate constant. The causes for a broad RTD are versatile, in microreactors mainly originating in flow maldistribution at 
the inlet and outlet and channel-to-channel non-uniformity. In the process of numbering-up or scaling-up attention needs to be paid to flow distribution. To minimize void volume the expansion angle between inlet and the reaction chamber is typically larger than $40^{\circ}$ (11). However, without any distribution features this will lead to recirculation zones or jetting. Consequently, a distributor at the inlet and a collector at the outlet are required. A range of different distributor designs is described in literature, focusing on equidistribution of the flow rate, minimal dispersion, minimal void volume and minimal pressure drop. A porous frit for instance will typically give a good equidistribution, preventing jetting, but comes with a high pressure drop. Other types of distributors are: Z-type arrangement (12), (13), perforated grids or baffles (11), branched or bifurcating networks (14-19) and radially interconnected networks (20). Which distributor is best suited, depends on the application. Bifurcating and radially interconnected distributors for instance will yield a narrower RTD than the Z-type distributor but comes with a higher pressure drop and are sensitive to fabrication errors (21).

Channel-to-channel non-uniformity can originate from production errors and also fouling is often unavoidable, both having the potential to change the reactor performance dramatically. Deviations of only a few $\mu \mathrm{m}$ on channels of $100-1000 \mu \mathrm{m}$ can have a severe impact on the flow distribution, making it difficult to achieve a homogeneous flow distribution with microchannel arrays. Wibel et al (2013) (4) calculated the impact of a geometric deviation $(\mathrm{g} \%)$ on the flow distribution $\left(\mathrm{u}_{\text {fast }} / \mathrm{u}_{\text {slow }}\right)$ between two channels as follow (Eq. 10):

$$
\frac{u_{\text {fast }}}{u_{\text {slow }}}=(1+g \%)^{2}
$$

Accordingly, to determine the approximate impact of varying parallel tubular channels, the Bodenstein number can be determined from the relative variance in residence time $\left(\hat{\sigma}_{t}^{2}\right)$ or channel diameter $\left(\hat{\sigma}_{d}^{2}\right)($ Eq. 11) (22):

$$
B o \approx \frac{2}{\hat{\sigma}_{t}^{2}} \approx \frac{1}{2 \hat{\sigma}_{d}^{2}}
$$

With this approximation it was derived that for a multi-channel microreactor with assumed plug flow behavior and first order kinetics the conversion drops e.g. $5 \%$ if there is a deviation of $10 \%$ on the channel diameter for 
heterogeneous first order reactions and for consecutive reactions it drops $4 \%$ at the optimum if the channel diameter varies $10 \%(22)$.

Besides parallelization, upscaling, often in one dimension (e.g. parallel plate configuration), can be performed to increase the reactor volume preferentially without leaving the $\mu \mathrm{m}$-scale. However, this will often result in a larger axial dispersion (23). Keeping the channel width or depth constant the axial dispersion in a rectangular duct (Fig. 2 a) is minimal with an aspect ratio (A) of 1. Increasing the aspect ratio will increase the axial variance maximum by roughly a factor of 4 , this at the parallel plate configuration with side wall effect. To minimize the axial dispersion Callewaert et al. (2014)(23) studied different cross-sectional designs. With an optimized pinched (P) cross-sectional design in the middle of the channel (Fig. 2 b), the axial dispersion at different aspect ratios is always smaller than with a rectangular cross-section of identical aspect ratio. At an aspect ratio of 1 the axial dispersion is even a little bit smaller than in the theoretical case of infinite parallel plate configuration without side walls, this by pinching the channel so far that two triangular channels are formed eliminating one corner. The axial dispersion is then more or less a factor 2 smaller in comparison to a quadrangular cross-section. At high aspect ratios (45 or higher) with an optimal pinched cross-section the axial dispersion is a factor 2.2-2.3 lower than with a rectangular cross-section. However, at an aspect ratio of 8 it becomes more beneficial to use an I-shaped crosssection (Fig. 2 c) than a pinched one. Hence, at an aspect ratio of 45 or higher the axial dispersion with an optimal I-shaped cross-section ( $\mathrm{W}$ and $\mathrm{H}$ ) is a factor of 7-8 lower, than a rectangular cross-section and a factor of 3-3.5 than a pinched one. Although the optimal pinched and I-profile result in a reduced axial dispersion, caution should prevail, as these lower axial dispersion coefficients only occur when those optimal geometrical parameters are achieved (23). For instance, fabrication errors will inevitably result in geometrical parameters $(P, H, W)$ that deviate from their optimum value. As a result, the axial dispersion can quickly become larger than with the rectangular cross-section. Besides changing the cross-sectional design, the topological channel design can also result in a narrower RTD. Meandering channels can give rise to secondary flow patterns and in some cases Dean vortices, which can reduce the broadening of residence time distributions, improve mixing and enhance heat transfer significantly (24).

When a heterogeneous catalyst is present the walls of microchannels can in some cases provide enough surface area. However, additional specific surface is generally needed (25). Either the channel wall can then be rendered

This article is protected by copyright. All rights reserved. 
porous or a packing can be inserted in the microchannel. With an improper packing design this can however lead to a serious broadening of the RTD, but it can also give an opportunity to narrow the RTD as Hu et al. (2014)(26) demonstrated. While an initially empty microreactor $(0.15 \times 0.15 \times 340 \mathrm{~mm}, 1.48-14.8 \mathrm{~mm} / \mathrm{s}, 0.3-3 \mathrm{~min})$ produced a Bodenstein number of $\mathrm{Bo}=52, \mathrm{Bo}=229$ was observed after packing the microreactor with quartz particles of 20 to $40 \mu \mathrm{m}$. This is due to the fact that the parabolic flow profile is 'broken'. Besides the spacing between the particles or support structures, also the uniformity of the locally formed channels determines the extent of dispersion. Packed bed and monolithic columns are well established formats to reduce the effective channel dimensions, but these are disordered systems. Monolithic columns typically require lower back pressures, but produce more dispersion (27). To combine the best of both worlds pillar array columns were introduced a decade ago (28). The non-contact nature and sheer unlimited freedom in structure placement of pillar arrays enables much smaller fluidic resistances, while the perfect order entirely eliminates eddy dispersion. Microfabrication furthermore offers the ability to introduce tailor made (anisotropic) shapes that can reduce dispersion even more. It was indeed shown recently (29) that diffusion originating axial dispersion (so-called Bterm, representing the axial dispersion contribution that is purely due to diffusion and is proportional to the molecular diffusion coefficient) could be reduced by more than an order of magnitude by elongating the support structures (Fig. 3) in the lateral (non-axial) direction by a ratio of more than 10 . These ordered pillar array structures can additionally be made porous (Fig. 4) to further increase the surface area, either by growing a layer onto the pillars (outwards direction) or making the pillar itself porous (inwards direction). Different types of layers can be grown outwards onto the pillar: zeolites, carbon nanotubes, carbon nanofibers and sol-gel. Describing these techniques into detail falls out of the scope of this review paper and the reader is referred to a previous review paper of our group (7). Growing a porous shell onto the pillars however constitutes as drawback that the initial topology, which can be controlled to just a few micrometer using etching techniques, changes and as a result yields a more irregular design. This can potentially enlarge the RTD. Sol-gel deposition is however an exception. It can yield both thin $(<10 \mu \mathrm{m})$ layers as ordered 3D networks in the presence of a pillar array and under specific conditions (pillar distance < domain size) (Fig. 5) (30). Growing the porous layer inwards, the original topology is not altered, maintaining the micrometer precision. For example electrochemical anodization can be employed for this (31) (32).

This article is protected by copyright. All rights reserved. 
Besides increasing the surface area for catalyst coating, a heterogeneous coating itself can also lead to a broader RTD. Delsman et al. (2005)(22) studied the impact of a spread in catalyst coating over parallel channels on RTD and conversion. When catalyst is unevenly divided over the parallel channels it will result in different catalyst coating thicknesses and lead to similar effects as emerging from a difference in channel diameter. For example, the reaction of oxygen with carbon monoxide and hydrogen leads to poisoning of the catalyst and when the catalyst is then 10 times more unevenly distributed over the channels, Delsman et al. (2005) (22) saw a drop in conversion of $10 \%$ already after 4 hours. A conformal coating is therefore crucial for a high efficiency. A large number of techniques are available to coat the surface of the channel (7). (33). Often an oxide support is first deposited which is subsequently impregnated with catalyst. Depending on the desired thickness different techniques are suitable: physical vapor deposition $(<1 \mu \mathrm{m})$, sol-gel deposition $(<10 \mu \mathrm{m})$ or suspension methods $(1-100 \mu \mathrm{m})$. Also the geometry of the surface influences the choice in deposition technique. For instance, when a porous surface needs to be coated, suspension is mostly not suited as the pores will be blocked.

A special operating condition is the periodic or unsteady state operation of continuously operated chemical reactors, which can lead to a considerable increase of selectivity and reactor performance compared to the optimal steady state condition (34). In periodic operation one or more operating parameters are continuously cycled (e.g. pulse injection of reagents). For instance, in the catalytic dehydration of an alcohol an adsorbed intermediate complex at the catalytic surface as well as a free site is needed for the reaction to proceed. Because of this the reaction rate increases considerably when the feed concentration decreases. By periodically varying the feed concentration a higher average reactor performance can hence be obtained (25). It is evident that in such an operation condition a narrow RTD is critical, which becomes more and more important as the ratio of cycle period over residence time decreases.

One application in which a narrow RTD is especially crucial, is particle synthesis. Most frequently this is performed in batch reactors (35), despite some serious drawbacks: insufficient mixing and heterogeneous distribution of reactants and temperature, which lead to broad particle-size distributions. Using a microreactor, consisting of a micromixer and reaction tubing, Gutierrez et al. (2011) (35) produced silica nanoparticles according to the Stöber synthesis process, that is often quoted as "one of the easiest to control nanoparticle

This article is protected by copyright. All rights reserved. 
preparation procedures". Nonetheless, they demonstrated the production of nanoparticles with a polydispersity which was $26 \%$ lower in comparison to batch experiments. Even in microreactors the laminar flow profile leads to dispersion, due to a difference in velocity near the wall and the center of the channel. This can lead to a higher particle size spread, even worse than batch as demonstrated by Khan et al. (2004) (36). To resolve this dispersion effect they introduced an inert gas to create slugs. Because of the physical boundary and the intense recirculation inside the slugs, this dispersion was avoided, resulting in silica particles with a size distribution equivalent to small-scale batch results.

\section{Combined reaction and separation}

When dealing for instance with equilibrium reactions or reactions with a high risk to form by-products (e.g. consecutive reactions), it is interesting to continuously remove the formed product. For this the downstream process and reactor unit have to be merged into one single unit. Consequently, it is crucial that the reactor has plug flow behavior, as it is vital to successful combine reaction and separation. A classic example is reactive distillation. However as this process consumes a lot of energy, alternatives are investigated.

\subsection{Chromatographic reactors}

Thinking about joined separation and reaction, chromatography columns have some interesting properties as the packing material that is used as support for the stationary phase for separation based on different adsorption affinities, is also suited as catalyst support.

Chromatographic reactors are mainly used for equilibrium-limited reactions (37). The simplest method is to discontinuously inject a pulse of reactants of a reversible reaction into a packed bed reactor. Because of the different affinities of the products for the packing inside the reactor the products are separated from one another while moving through the bed. This prevents the reversed reaction from taking place, shifting the equilibrium to the product side.

This article is protected by copyright. All rights reserved. 
The low yields and high product dilution states present however serious drawbacks. To tackle these drawbacks processes which operate continuously were developed. An example of this is simulated moving bed (SMB) chromatography (Fig. 6), patented in 1961 by Universal Oil Products (37).

In SMB the feed is continuously added while the particles move in opposite direction, creating a counter-current flow. Due to this counter-current flow, separation is obtained, as the strongly retained molecules will adsorb on the particles and dragged along, while the weakly retained molecules remain in the liquid flow. To desorb the strongly retained molecules a desorbing liquid is fed somewhere in between the raffinate and extract collection point. A true counter-current flow of the liquid and the particles is not easily obtained, related to the difficulty of reliably handling dense slurries of particles. A work-around is to use multiple columns and periodically switch the position of the feed and desorbent inlets and extract and raffinate outlets between the columns, which is equivalent to a true moving bed; hence the name simulated moving bed.

An integrated reaction and separation system is not always desirable. For instance, in SMB zones can exist where reactant is enriched, which drives equilibrium reactions to the desired direction, but also zones with enriched product exist which could drive the reaction in the opposite direction (38).

Highly interesting systems for combined reaction and separation are enzymatic reactions. Due to inhibition, either by a substrate or a formed product, enzymatic reactions often results in a low yield. Because of this, either removing the formed product or dosing the substrate is necessary. Often, this is performed consecutively with an enzymatic reactor followed by a separation, in which the non-converted substrate is recycled. Typically, this can be performed with SMB. However, as the effluent flows are usually larger than the feed flow only a fraction can be recycled unless solvent is removed, e.g. by nanofiltration (38). This can be omitted by integrating reaction and separation. Instead of using SMB consecutively after a reactor unit, it can also act as a reactor itself. The enzyme can either be continuously added through the feed stream or immobilized on the solid phase. For instance, by continuously removing the product galactose, which strongly inhibits the enzyme lactose, with SMB, only $32-51 \%$ of the amount of enzyme used in batch operation was needed to obtain the same yield (39). However, for such a system to work, the solvent used should be at the same time suited as reaction medium as mobile phase. Because of this, often an ion-exchange packing is used as it allows water-based mixtures as effluent. Also

This article is protected by copyright. All rights reserved. 
reversed phase silica coatings can be used, but the enzyme must then tolerate small amounts of organic solvent, as reversed phase coatings are not compatible with pure water solutions (38).

\subsection{Membrane reactors}

Equipping the (micro)reactor with a membrane likewise allows to selectively remove one of the products and additionally gives the possibility to dose one of the reactants at the position where it is required (40). Employing microreactor technology high surface to volume ratios are obtained, thereby considerably increasing the efficiency. Lai et al. (2003) (41) and Zhang et al. (2004) (42) performed the Knoevanagel condensation reaction inside a membrane microreactor (MMR) with parallel microchannels, reaching supra-equilibrium conversions. In this reaction water is a byproduct, limiting the yield. By growing a hydrophilic zeolite membrane on the porous steel plate, containing the microchannels (Fig. 7), they were able to continuously remove the formed water, increasing the yield with $40 \%$. Besides this plate-type configuration also the hollow fiber (or tubular) configuration is often used. Yamamoto et al. (2006) (43) constructed a tubular membrane microreactor consisting of a $\alpha$ alumina porous tube coated on the shell side by chemical vapor deposition with a palladium membrane (Fig. 8). In the lumen side cyclohexane was dehydrogenated to benzene and the formed hydrogen was removed through the membrane which was only permeable for hydrogen, shifting the equilibrium. As the $\alpha$-alumina porous tube had a large internal diameter of $1.6 \mathrm{~mm}$, different stainless steel rods with varying diameter size were inserted at the lumen side to decrease the gap towards the membrane. By decreasing this gap both conversion as selectivity towards benzene increased, possibly due to a more uniform temperature profile and larger supply of reactant to the catalyst. Advantageous of the hollow fiber is that it is self-supporting, omitting the need for a support layer. Such a support layer is rapidly a few hundred $\mu \mathrm{m}$ up to a few millimeters thick, lowering the surface to volume ratio. However, because they are self-supporting, the mechanical stability is low, limiting the maximal pressure difference. Instead of removing one of the products, (micro)reactors equipped with a membrane can be used to contact two phases without mixing $(44,45)$. With membrane (micro)reactors, a high contact surface (higher than in batch) (46) can be obtained and potential hazardous situations (e.g. hotspots or runaways) can be avoided, just as the formation of unwanted by-products (47). Park et al. (2010) (46) compared the Heck reaction in a batch reactor and membrane microreactor (Fig. 9). As the catalyst palladium has to be reactivated with oxygen, a high contact surface is crucial. Due to a higher contact surface the selectivity was much higher in the membrane

This article is protected by copyright. All rights reserved. 
microreactor (7.7:1 - 13.7:1) than in batch (2.7:1 - 2.9:1). van Gool et al. (2013) (48) studied the difference between presaturating the reaction streams with $\mathrm{CO}_{2}$ and in situ dosing of $\mathrm{CO}_{2}$ using a membrane microreactor. By continuously diffusing $\mathrm{CO}_{2}$ in the reaction stream (membrane microreactor) a nearly full conversion was obtained, while with preconcentrating the reaction stream the conversion was only $90 \%$. Although, it should be mentioned that the latter system was more robust, as clogging was no longer an issue and the temperature could be regulated.

To create a stable parallel flow profile a membrane is not necessary (49). Omitting a membrane significantly reduces the mass transfer resistance from minutes of needed residence time (44) to tens of seconds $(50,51)$. However, by operating without a membrane the operating range where the parallel flow is stable, expressed through the breakthrough pressure, reduces from a few bar (52) to only $1000 \mathrm{~Pa}(53)$, constituting a large disadvantage.

\subsection{Electrophoretically mediated microanalysis}

Combined reaction and separation is in first instance interesting to increase the yield, but the concept also offers additional advantages. To reduce the sample size or amount of reagent, it is interesting to combine the reaction and separation step such that sample preparation and analysis can occur on a single chip. To obtain a high separation efficiency, miniaturization is often necessary. Electrophoretically mediated microanalysis (EMMA) (5456) is an example of this, which was first described by Bao and Regnier (1992) (57).

In EMMA reagents are mixed in a capillary with a typical diameter in the range of tens of $\mu \mathrm{m}$, subsequently the formed products are separated from the remaining reagents by electrophoresis. This technique is typically used to study the activity of enzymes, as it requires only small amounts of the often expensive enzymes and/or substrates. Two main operation modes of EMMA are reported in the literature: continuous and plug-plug mode (Fig. 10).

In the continuous mode the capillary is completely filled with the first reagent and the second reagent is either introduced as a plug (= zonal sample introduction) or continuously fed at the inlet (= moving boundary condition).

This article is protected by copyright. All rights reserved. 
In the moving boundary condition the second reagent has to migrate faster through the capillary than the first reagent by electrophoresis in order to mix the reagents, which is not necessary in the zonal sample introduction method. However the reactant overlap is greater in the moving boundary condition method, resulting in a concentration sensitivity which is an order of magnitude higher. To further minimize the sample size a second mode, the plug-plug mode, was developed. In this mode sample bands of reagent one and two are consecutively injected. Both plugs are subsequently mixed and the formed products and reagents are separated as the voltage is turned on. To mix the plugs different approaches are used. In the classical mode the second plug has a higher migration speed than the first one and thus passes through this first plug. Alternatively, both plugs are mixed at the first section near the inlet of the capillary by diffusion, allowing to mix more than two plugs, which is also possible by using an electrical field but is considered difficult. Another advantage of the plug-plug mode over the continuous mode is that before and after the plugs of reagent a plug containing a buffer at a different $\mathrm{pH}$ can be added. Often the $\mathrm{pH}$ in the background electrolyte needed for the separation is not compatible with the $\mathrm{pH}$ needed for the reaction. Adding the buffer plugs allows the reaction to proceed in the first section of the capillary and subsequently in the remaining part of the capillary the product and reagents are separated. With plug-plug mode the obtained electropherograms are also comparable to those obtained with classical capillary electrophoresis and thus the analysis can be performed by classical integration software.

In a special case of EMMA capillary electrophoresis is combined with an enzyme microreactor. Instead of injecting a plug of enzyme, it can be immobilized inside the capillary channel. Yan et al. (2010) (58) coated magnetic microbeads of $2.8 \mu \mathrm{m}$ with alkaline phosphatase which were held in place with a single magnet placed $350 \mu \mathrm{m}$ or two magnets placed $395 \mu \mathrm{m}$ from the center of the capillary with an internal diameter of $50 \mu \mathrm{m}$. The entire channel was then filled with the fluorogenic substrate AttoPhos. In this way the peak capacity was increased, allowing to measure multiple reversible inhibitors in one single run as the inhibitors are separated by capillary electrophoresis before reaching the immobilized magnetic beads coated with enzyme. Immediately after the enzyme beads the concentration of a fluorescent product is measured, resulting in negative peaks when an inhibitor plug passes through the enzyme bed.

EMMA uses electroosmotic flow (EOF) for transporting the introduced plugs. This is however not always possible as EOF requires a dissolved electrolyte. To circumvent this problem pressure-mediated microanalysis (PMMA)

This article is protected by copyright. All rights reserved. 
was developed. In PMMA the flow is obtained by inducing a pressure gradient, as a drawback the plugs than have to be mixed based purely on diffusion.

\subsection{Emerging opportunities}

Thanks to the enormous freedom that miniaturized reactors offer in terms of dispersion, (integrated) separations and optimized energy housekeeping new developments can be anticipated in the near future. The thermodynamic 'branch' of PI is e.g. often associated with the local incorporation of energy sources (59) and alternative driving forces (60), whereby a smart reactor design and implementation of the energy source can provide energy at the place where it is useful, i.e. at the area where reagents are contacted and where the reaction actually takes place. Considering that the fraction of the energy that is provided to solvent and already formed products is lost, it is clear that this branch should be approached from a much broader perspective. While it is well known that the equilibrium product concentration of an equilibrium limited reaction can be dramatically increased when the product is instantaneously removed, the opportunities (and challenges) that emerge when combining this feature with a local definition of reaction sites and energy provision are hardly addressed in the literature. Prominent examples of existing approaches to combine separation and reaction are reactive distillation (61) and reactive simulated moving bed chromatography (62). While these methods are extremely valuable and still in full development, they do not allow for selective energy housekeeping. In order to achieve spatially well distinct and accessible zones, 2D separation techniques involving an electrical (or magnetic) force field (63) could e.g. be perfectly suited for this goal.

Enantiomeric reactions are a special case that could benefit highly from combined reaction and separation as they mostly result in a racemic mixture ( $50 \%$ R-enantiomer and $50 \%$ S-enantiomer), limiting the yield to a theoretical maximum of $50 \%$. To surpass this limit the unwanted enantiomer has to be converted again to a racemic mixture without converting the wanted enantiomer. Because of this, reaction and separation are currently only performed consecutively. Not only to increase the yield, but also the demand to pure enantiomers is growing from industry, in particular in the pharmaceutical industry, where the opposite enantiomer is sometimes toxic. A well-known and successful approach to separate enantiomers is crystallization. However, to obtain a pure

This article is protected by copyright. All rights reserved. 
enantiomer, the feed composition must lie past the eutectic point. Simply removing a part of the solvent by evaporation, to pass the solubility curve, is sometimes not enough to reach the desired purity (64). A second approach is to use preparative chromatography to separate the enantiomers, either (semi-) discontinuously (e.g. annular chromatography) or continuously (e.g. SMB). However, as the enantiomers only differ in their geometrical molecular structure they are physicochemically very alike and expensive chiral stationary phases with a high loading capacity and selectivity are necessary, resulting in a high cost. To decrease this cost a hybrid process has been described (64-67). First a chromatographic separation is performed, to pre-concentrate the feed, surpassing the eutectic point. Once passed, the enriched mixtures can be crystallized, resulting in the pure enantiomer. However, with this hybrid process the chromatographic separation is indeed less crucial. Nonetheless, some sort of chromatographic separation is still needed, requiring a suitable chiral agent (stationary phase). To omit the need for such chiral agents, Howard et al. (1976) (68) was the first to propose the idea to separate enantiomers by hydrodynamics. Due to chirality the two enantiomers rotate in a different manner, by imposing an asymmetrical flow profile this then leads to a different chiral-specific average velocity (69) (Fig. 11). Aristov et al. (2013) (70) demonstrated this experimentally by separating a plug of chiral colloidal particles $(6 \times 6 \times$ $6 \mu \mathrm{m})$. Periodical grooves were created in the top of a rectangular microchannel (150 $\mu \mathrm{m} \times 115 \mu \mathrm{m})$, resulting in a helical asymmetrical flow. As particles always prefer those positions where their frictional losses are minimal, it resulted in a different spatial position in the channel, leading to a different average velocity. Such separations have at the moment only been demonstrated for chiral particles $(71,72)$. To separate enatiomeric molecules the chiral specific drift must be larger than the Brownian motion. Hermans et al. (2015) (71) described and demonstrated this chiral specific motion, allowing to estimate the required minimal particle size. With this Couette cell particles should be in the range of $10-100 \mathrm{~nm}$, to allow a proper separation. Otherwise, the chiral drift vanishes due to Brownian motion. This minimal size lies far beyond the size of small enantiomeric molecules (1$0.1 \mathrm{~nm})$. To overcome the Brownian motion for small enantiomeric molecules most likely nanoscale dimensions are necessary, requiring innovative solutions especially if it is to be integrated with the reactor unit.

This article is protected by copyright. All rights reserved. 


\section{Concluding remarks}

In order to take one of the next steps in PI, i.e. near plug flow behavior and combined reaction and separation, microreactors due to their small channel dimensions, yielding fast heat and mass transfer, have a high potential. However, to achieve industrially relevant throughputs, numbering-up or scaling-up is necessary. Attention then needs to be paid to the flow distribution, channel design and packing in order to prevent dispersion. Chromatographic systems can be used as an inspiration as they excel in minimal dispersion and high separation factors. For instance using elongated pillars will yield high surface to volume ratios while keeping dispersion to a minimum. Integrating catalyst or enzymes in such systems with low RTD consequently results in high efficiency gains, as side-product formation or inhibition can be avoided. As reduced dispersion will allow for integrated separation it also allows to locally administer reactants (e.g. for catalyst regeneration), while at the same time it

can also lead to local energy housekeeping. A special case that could benefit highly from integrated reaction and separation are enantiomeric products. While preliminary hydrodynamic separation principles have been demonstrated, no integrated system has been reported so far.

\section{Acknowledgements}

W. De Malsche greatly acknowledges the Flemish Fund for Scientific Research (FWO) for support through a Post-Doctoral grant. J. Hereijgers is supported through a specialization grant from the Instituut voor Wetenschap en Technologie (IWT) from the Flanders region.

The authors have declared no conflict of interest

This article is protected by copyright. All rights reserved. 
Figures

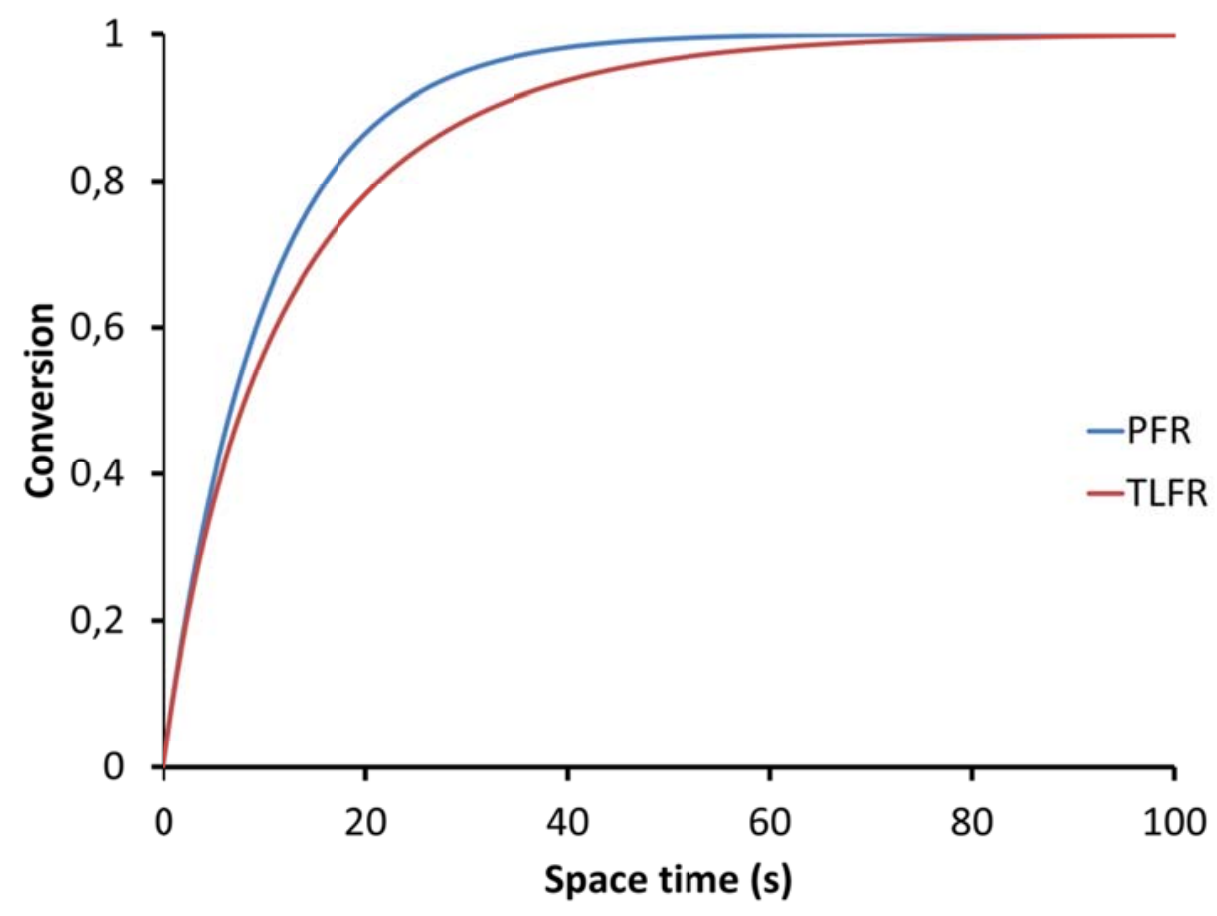

Fig. 1: Conversion in function of the space time for a first order reaction $\left(k=0.1 \mathrm{~s}^{-1}\right)$ in an ideal plug flow reactor and tubular laminar flow reactor.

This article is protected by copyright. All rights reserved. 

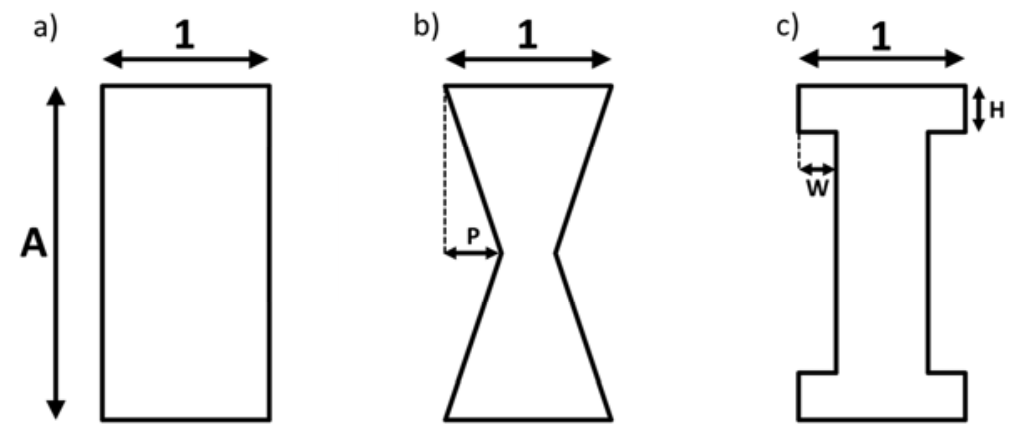

Fig. 2: Cross sectional view of a a) rectangular profile b) pinched profile c) I-profile.

This article is protected by copyright. All rights reserved. 


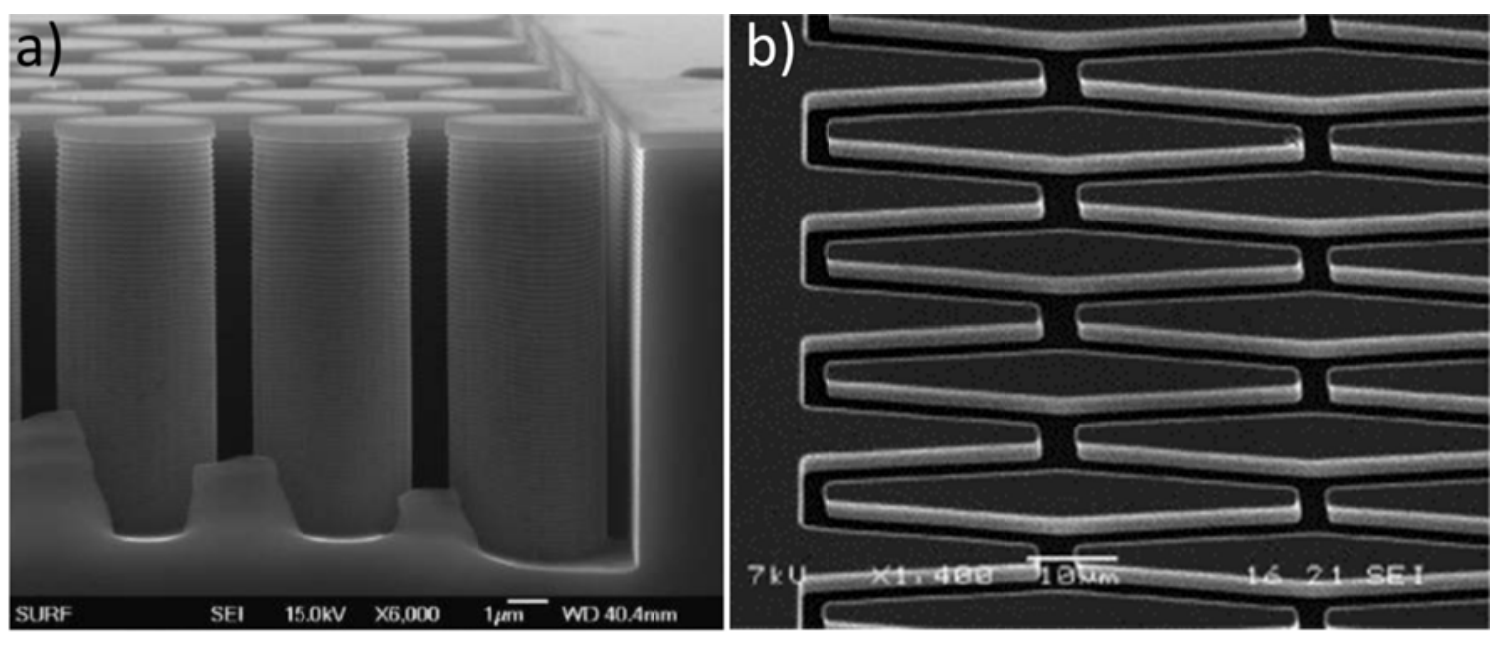

Fig. 3: a) circular array pillar bed (Reprinted from (73)), b) radially elongated array pillar bed (Reprinted from (74) with permission from Elsevier). 


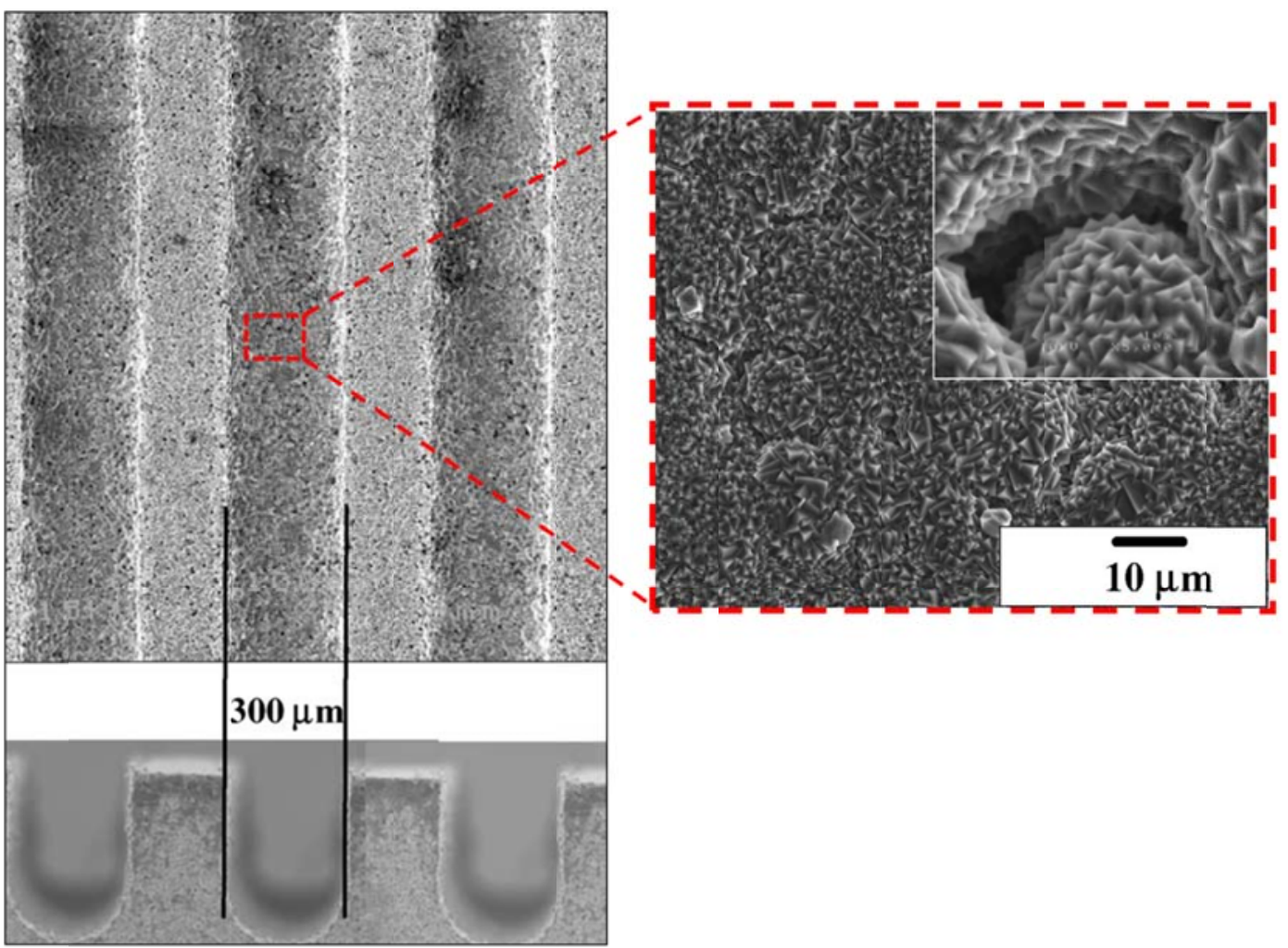

Fig. 7: Deposition of a hydrophilic zeolite membrane onto porous steel channels. Reprinted from (42) with permission from Elsevier.

This article is protected by copyright. All rights reserved. 


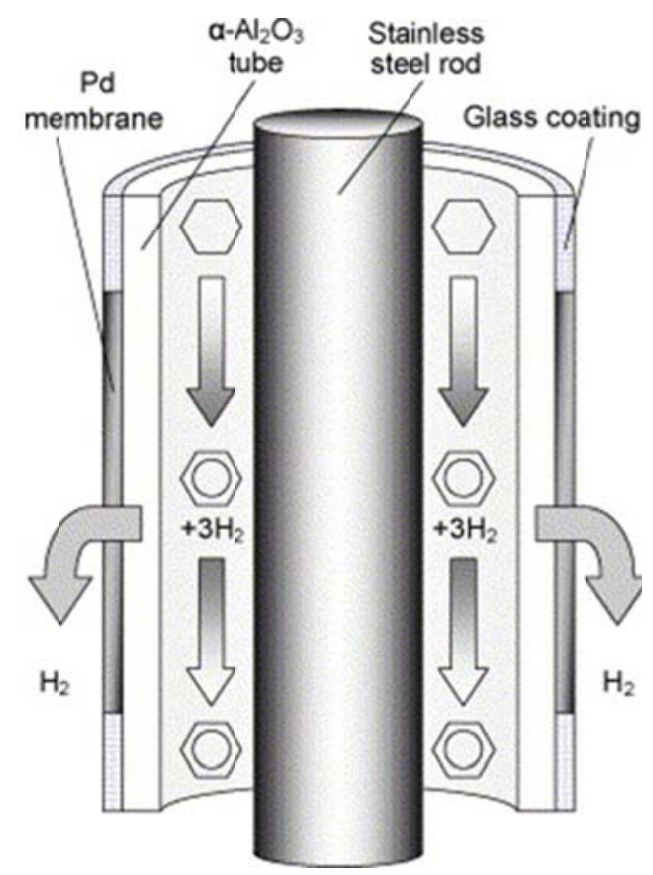

Fig. 8: Tubular membrane microreactor coated with palladium at the outer $\alpha-\mathrm{Al}_{2} \mathrm{O}_{3}$ porous shell. Reprinted from (43) with permission from Elsevier.

This article is protected by copyright. All rights reserved. 


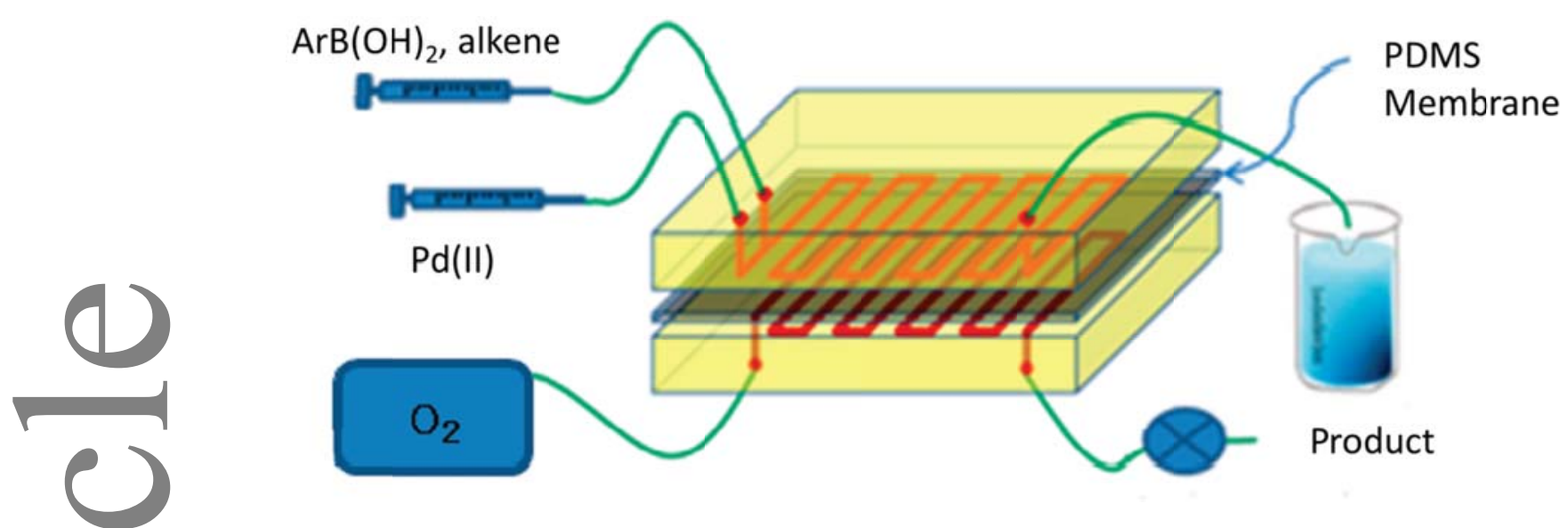

Fig. 9: Membrane microreactor. Reprinted with permission from (46). Copyright 2015 American Chemical Society.

This article is protected by copyright. All rights reserved. 


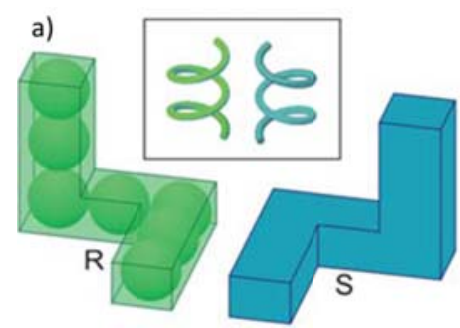

b)
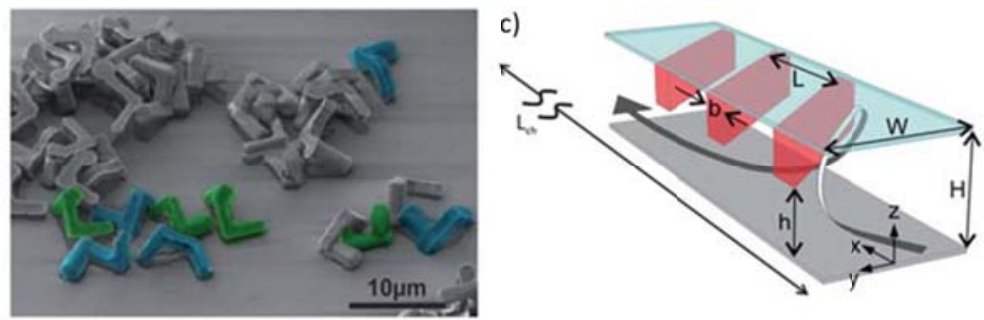

Fig. 11: a) Schematic illustration of particles with opposite chirality. b) SEM picture of the chiral particles with some particles artificially colored. c) Microfluidic channel with helical flow field. Reproduced from Ref. (70) with permission from The Royal Society of Chemistry.

This article is protected by copyright. All rights reserved. 


\section{References}

1. Lomel S, Falk L, Commenge JM, Houzelot JL, Ramdani K. The Microreactor: A Systematic and Efficient Tool for the Transition from Batch to Continuous Process? Chem Eng Res Des [Internet]. 2006 May [cited 2015 Jun 21];84(5):363-9. Available from:

http://www.sciencedirect.com/science/article/pii/S0263876206729069

2. Yoshida J, Takahashi Y, Nagaki A. Flash chemistry: flow chemistry that cannot be done in batch. Chem Commun [Internet]. The Royal Society of Chemistry; 2013 Nov 4 [cited 2015 Jun 21];49(85):9896-904. Available from: http://pubs.rsc.org/en/content/articlehtml/2013/cc/c3cc44709j

3. Levenspiel O. Chemcial Reaction Engineering. 3th ed. New York, NY: John Wiley \& Sons; 1999.

4. Wibel W, Wenka A, Brandner JJ, Dittmeyer R. Reprint of: Measuring and modeling the residence time distribution of gas flows in multichannel microreactors. Chem Eng J [Internet]. Elsevier B.V.; 2013;227:203-14. Available from: http://dx.doi.org/10.1016/j.cej.2013.05.016

5. Taylor G. The Dispersion of Matter in Turbulent Flow through a Pipe. Proc R Soc A Math Phys Eng Sci. 1954;223(1155):446-68.

6. Aris R. On the Dispersion of a Solute in a Fluid Flowing through a Tube. Proc R Soc A Math Phys Eng Sci [Internet]. 1956 Apr 10 [cited 2015 Jul 1];235(1200):67-77. Available from: http://rspa.royalsocietypublishing.org/content/235/1200/67

7. Hereijgers J, Desmet G, Breugelmans T, De Malsche W. Strategies to integrate porous layers in microfluidic devices. Microelectron Eng [Internet]. Elsevier B.V.; 2015 Jan;132:1-13. Available from: http://linkinghub.elsevier.com/retrieve/pii/S0167931714004262

8. Goovaerts R, Smits W, Desmet G, Denayer J, De Malsche W. Combined improved mixing and reduced energy dissipation by combining convective effects and lamination. Chem Eng J [Internet]. Elsevier B.V.; 2012;211-212:260-9. Available from: http://dx.doi.org/10.1016/j.cej.2012.09.024

9. Bhagat AAS, Peterson ETK, Papautsky I. A passive planar micromixer with obstructions for mixing at low Reynolds numbers. J Micromechanics Microengineering [Internet]. 2007 May 1 [cited 2015 Jun 30];17(5):1017-24. Available from: http://stacks.iop.org/0960-1317/17/i=5/a=023

10. Fogler HS. Elements of Chemical Reaction Engineering (4th Edition). New Jersey: Prentice Hall; 2005.

11. Rebrov E V., Schouten JC, de Croon MHJM. Single-phase fluid flow distribution and heat transfer in microstructured reactors [Internet]. Chemical Engineering Science. Elsevier; 2011. p. 1374-93. Available from: http://dx.doi.org/10.1016/j.ces.2010.05.044

12. Wang J. Pressure drop and flow distribution in parallel-channel configurations of fuel cells: Z-type arrangement. Int J Hydrogen Energy [Internet]. Elsevier Ltd; 2010;35(11):5498-509. Available from: http://dx.doi.org/10.1016/j.ijhydene.2010.02.131

13. Commenge JM, Falk L, Corriou JP, Matlosz M. Optimal Design for Flow Uniformity in Microchannel Reactors. AlChE J. 2002;48(2):345-58.

14. Luo L, Tondeur D, Le Gall H, Corbel S. Constructal approach and multi-scale components. Appl Therm Eng. 2007;27(10):1708-14.

This article is protected by copyright. All rights reserved. 
15. Luo L, Tondeur D. Optimal distribution of viscous dissipation in a multi-scale branched fluid distributor. Int J Therm Sci [Internet]. 2005 Dec [cited 2013 Mar 21];44(12):1131-41. Available from:

http://linkinghub.elsevier.com/retrieve/pii/S1290072905002139

16. Fan Z, Zhou X, Luo L, Yuan W. Numerical Investigation of Constructal Distributors with Different Configurations. Chinese J Chem Eng [Internet]. Chemical Industry and Engineering Society of China (CIESC) and Chemical Industry Press (CIP); 2009 Feb [cited 2013 Apr 12];17(1):175-8. Available from: http://linkinghub.elsevier.com/retrieve/pii/S1004954109600525

17. Luo L, Fan Y, Zhang W, Yuan X, Midoux N. Integration of constructal distributors to a mini crossflow heat exchanger and their assembly configuration optimization. Chem Eng Sci [Internet]. 2007 Jul [cited 2015 Jun 5];62(13):3605-19. Available from:

http://www.sciencedirect.com/science/article/pii/S0009250907002825

18. Tondeur D, Luo L. Design and scaling laws of ramified fluid distributors by the constructal approach. Chem Eng Sci [Internet]. 2004 Apr [cited 2013 Mar 4];59(8-9):1799-813. Available from:

http://linkinghub.elsevier.com/retrieve/pii/S0009250904000934

19. Vangelooven J, De Malsche W, Op De Beeck J, Eghbali H, Gardeniers H, Desmet G. Design and evaluation of flow distributors for microfabricated pillar array columns. Lab Chip [Internet]. 2010 Feb 7 [cited 2013 Mar 17];10(3):349-56. Available from: http://www.ncbi.nlm.nih.gov/pubmed/20091007

20. Vangelooven J, Desmet G. Computer aided design optimisation of microfluidic flow distributors. J Chromatogr A [Internet]. Elsevier B.V.; 2010;1217(43):6724-32. Available from:

http://dx.doi.org/10.1016/j.chroma.2010.05.032

21. Davydova E, Deridder S, Eeltink S, Desmet G, Schoenmakers PJ. Optimization and evaluation of radially interconnected versus bifurcating flow distributors using computational fluid dynamics modelling. J Chromatogr A [Internet]. 2015 Feb 6 [cited 2015 Jun 7];1380:88-95. Available from: http://www.sciencedirect.com/science/article/pii/S0021967314019955

22. Delsman ER, de Croon MHJM, Elzinga GD, Cobden PD, Kramer GJ, Schouten JC. The Influence of Differences Between Microchannels on Micro Reactor Performance. Chem Eng Technol [Internet]. 2005;28(3):367-75. Available from: http://doi.wiley.com/10.1002/ceat.200407126

23. Callewaert M, De Malsche W, Ottevaere H, Thienpont H, Desmet G. Assessment and numerical search for minimal Taylor-Aris dispersion in micro-machined channels of nearly rectangular cross-section. J Chromatogr A [Internet]. 2014 Nov 14 [cited 2015 Jul 1];1368:70-81. Available from: http://www.sciencedirect.com/science/article/pii/S0021967314013892

24. Aubin J, Prat L, Xuereb C, Gourdon C. Effect of microchannel aspect ratio on residence time distributions and the axial dispersion coefficient. Chem Eng Process Process Intensif. 2009;48(1):554-9.

25. Rouge a., Spoetzl B, Gebauer K, Schenk R, Renken a. Microchannel reactors for fast periodic operation: The catalytic dehydration of isopropanol. Chem Eng Sci. 2001;56(4):1419-27.

26. Hu C, Hartman RL. High-throughput packed-bed microreactors with in-line analytics for the discovery of asphaltene deposition mechanisms. AlChE J [Internet]. 2014 Oct 10 [cited 2015 Jul 1];60(10):3534-46. Available from: http://doi.wiley.com/10.1002/aic.14542

27. Ishizuka N, Kobayashi H, Minakuchi H, Nakanishi K, Hirao K, Hosoya K, et al. Monolithic silica columns for high-efficiency separations by high-performance liquid chromatography. J Chromatogr A [Internet]. 2002 Jun [cited $2015 \mathrm{Jul}$ 1];960(1-2):85-96. Available from: http://www.sciencedirect.com/science/article/pii/S0021967301015801

This article is protected by copyright. All rights reserved. 
28. De Malsche W, Eghbali H, Clicq D, Vangelooven J, Gardeniers H, Desmet G. Pressure-Driven ReversePhase Liquid Chromatography Separations in Ordered Nonporous Pillar Array Columns. Anal Chem [Internet]. American Chemical Society; 2007 Aug 1 [cited 2015 Jul 1];79(15):5915-26. Available from: http://dx.doi.org/10.1021/ac070352p

29. Op De Beeck J, Callewaert M, Ottevaere H, Gardeniers H, Desmet G, De Malsche W. On the Advantages of Radially Elongated Structures in Microchip-Based Liquid Chromatography. Anal Chem [Internet]. American Chemical Society; 2013 May 21 [cited 2015 Jul 1];85(10):5207-12. Available from: http://dx.doi.org/10.1021/ac400576s

30. Detobel F, Eghbali H, De Bruyne S, Terryn H, Gardeniers H, Desmet G. Effect of the presence of an ordered micro-pillar array on the formation of silica monoliths. J Chromatogr A. 2009;1216(44):7360-7.

31. Callewaert M, De Beeck JO, Maeno K, Sukas S, Thienpont H, Ottevaere H, et al. Integration of uniform porous shell layers in very long pillar array columns using electrochemical anodization for liquid chromatography. Analyst [Internet]. The Royal Society of Chemistry; 2014 Dec 24 [cited 2015 Jul 1];139(3):618-25. Available from: http://pubs.rsc.org/en/content/articlehtml/2014/an/c3an02023a

32. Losey MW, Jackman RJ, Firebaugh SL, Schmidt MA, Jensen KF. Design and fabrication of microfluidic devices for multiphase mixing and reaction. J Microelectromechanical Syst [Internet]. 2002 Dec [cited $2015 \mathrm{Jul} 1$ 1];11(6):709-17. Available from:

http://ieeexplore.ieee.org//pdocs/epic03/wrapper.htm?arnumber=1097791

33. Meille V. Review on methods to deposit catalysts on structured surfaces. Appl Catal A Gen. 2006;315:117.

34. Silveston P, Hudgins RR, Renken A. Periodic operation of catalytic reactors-introduction and overview. Catal Today [Internet]. 1995 Aug [cited $2015 \mathrm{Jul}$ 1];25(2):91-112. Available from: http://www.sciencedirect.com/science/article/pii/092058619500101K

35. Gutierrez L, Gomez L, Irusta S, Arruebo M, Santamaria J. Comparative study of the synthesis of silica nanoparticles in micromixer-microreactor and batch reactor systems. Chem Eng J [Internet]. Elsevier B.V.; 2011;171(2):674-83. Available from: http://dx.doi.org/10.1016/j.cej.2011.05.019

36. Khan S a, Günther A, Schmidt M a, Jensen KF. Microfluidic synthesis of colloidal silica. Langmuir. 2004;20(20):8604-11.

37. Rodrigues a. E, Pereira CSM, Santos JC. Chromatographic Reactors. Chem Eng Technol. 2012;35(7):1171-83.

38. Bechtold M, Makart S, Heinemann M, Panke S. Integrated operation of continuous chromatography and biotransformations for the generic high yield production of fine chemicals. J Biotechnol. 2006;124(1):14662.

39. Shieh MT, Barker PE. Combined bioreaction and separation in a simulated counter-current chromatographic bioreactor-separator for the hydrolysis of lactose. J Chem Technol Biotechnol. 1996;66(3):265-78.

40. De Jong J, Lammertink RGH, Wessling M. Membranes and microfluidics: a review. Lab Chip [Internet]. The Royal Society of Chemistry; 2006 Sep 23 [cited 2015 Jun 2];6(9):1125. Available from: http://pubs.rsc.org/en/content/articlehtml/2006/lc/b603275c

41. Lai SM, Ng CP, Martin-Aranda R, Yeung KL. Knoevenagel condensation reaction in zeolite membrane microreactor. Microporous Mesoporous Mater. 2003;66(2-3):239-52.

This article is protected by copyright. All rights reserved. 
42. Zhang X, Lai ESM, Martin-Aranda R, Yeung KL. An investigation of Knoevenagel condensation reaction in microreactors using a new zeolite catalyst. Appl Catal A Gen. 2004;261(1):109-18.

43. Yamamoto S, Hanaoka T, Hamakawa S, Sato K, Mizukami F. Application of a microchannel to catalytic dehydrogenation of cyclohexane on Pd membrane. Catal Today [Internet]. 2006 Oct [cited $2015 \mathrm{Jul}$ 1];118(1-2):2-6. Available from: http://www.sciencedirect.com/science/article/pii/S0920586106003166

44. Hereijgers J, Callewaert M, Lin X, Verelst $\mathrm{H}$, Breugelmans $\mathrm{T}$, Ottevaere $\mathrm{H}$, et al. A high aspect ratio membrane reactor for liquid-liquid extraction. J Memb Sci [Internet]. Elsevier; 2013 Feb [cited $2013 \mathrm{Mar}$ 5];436:154-62. Available from: http://linkinghub.elsevier.com/retrieve/pii/S0376738813001397

45. Cai ZX, Fang Q, Chen HW, Fang ZL. A microfluidic chip based liquid-liquid extraction system with microporous membrane. Anal Chim Acta. 2006;556(1):151-6.

46. Park CP, Kim D-P. Dual-Channel Microreactor for Gas-Liquid Syntheses. J Am Chem Soc [Internet]. American Chemical Society; 2010 Jul 28 [cited 2015 Jul 1];132(29):10102-6. Available from: http://dx.doi.org/10.1021/ja102666y

47. Dixon AG. Analysis of intermediate product yield in distributed-feed nonisothermal tubular membrane reactors. Catal Today [Internet]. 2001 May [cited 2015 Jul 1];67(1-3):189-203. Available from: http://www.sciencedirect.com/science/article/pii/S0920586101002875

48. Van Gool JJF, van den Broek SAMW, Ripken RM, Nieuwland PJ, Koch K, Rutjes FPJT. Highly Controlled Gas/Liquid Processes in a Continuous Lab-Scale Device. Chem Eng Technol [Internet]. 2013 Jun 13 [cited 2015 Jul 1];36(6):1042-6. Available from: http://doi.wiley.com/10.1002/ceat.201200553

49. Aota A, Mawatari K, Kitamori T. Parallel multiphase microflows: fundamental physics, stabilization methods and applications. Lab Chip [Internet]. 2009 Sep 7 [cited 2013 Mar 4];9(17):2470-6. Available from: http://www.ncbi.nlm.nih.gov/pubmed/19680572

50. Priest C, Zhou J, Klink S, Sedev R, Ralston J. Microfluidic Solvent Extraction of Metal lons and Complexes from Leach Solutions Containing Nanoparticles. Chem Eng Technol [Internet]. 2012 Jul 26 [cited 2015 Jun 30];35(7):1312-9. Available from: http://doi.wiley.com/10.1002/ceat.201100602

51. Priest C, Zhou J, Sedev R, Ralston J, Aota A, Mawatari K, et al. Microfluidic extraction of copper from particle-laden solutions. Int J Miner Process [Internet]. 2011 Mar [cited 2015 Jun 30];98(3-4):168-73. Available from: http://www.sciencedirect.com/science/article/pii/S0301751610001523

52. Hereijgers J, Breugelmans T, De Malsche W. Breakthrough in a flat channel membrane microcontactor. Chem Eng Res Des [Internet]. Institution of Chemical Engineers; 2015;94:98-104. Available from: http://linkinghub.elsevier.com/retrieve/pii/S0263876214005152

53. Priest C, Hashmi S, Zhou J, Sedev R, Ralston J. Microfluidic Solvent Extraction of Metal lons from Industrial Grade Leach Solutions: Extraction Performance and Channel Aging. J Flow Chem [Internet]. Akadémiai Kiadó; 2013 Sep 16 [cited 2015 Jun 30];3(3):76-80. Available from: http://www.akademiai.com/doi/abs/10.1556/JFC-D-13-00005?journalCode=1846

54. Wang X, Li K, Adams E, Schepdael A Van. Recent advances in CE-mediated microanalysis for enzyme study. Electrophoresis. 2014;35(1):119-27.

55. Nováková S, Van Dyck S, Van Schepdael A, Hoogmartens J, Glatz Z. Electrophoretically mediated microanalysis. J Chromatogr A [Internet]. 2004 Apr [cited 2015 Jul 1];1032(1-2):173-84. Available from: http://www.sciencedirect.com/science/article/pii/S0021967303023185

This article is protected by copyright. All rights reserved. 
56. Hai X, Yang BF, van Schepdael A. Recent developments and applications of EMMA in enzymatic and derivatization reactions. Electrophoresis. 2012;33(1):211-27.

57. Bao J, Regnier FE. Ultramicro enzyme assays in a capillary electrophoretic system. J Chromatogr A [Internet]. 1992 Sep 11 [cited 2015 Jul 1];608(1-2):217-24. Available from:

http://www.ncbi.nlm.nih.gov/pubmed/1430025

58. Yan X, Gilman SD. Improved peak capacity for CE separations of enzyme inhibitors with activity-based detection using magnetic bead microreactors. Electrophoresis. 2010;31(2):346-54.

59. Van Gerven T S a. Structure, energy, synergy, times the fundamentals of process intensification. Ind Eng Chem Res. 2009;48:2465-74.

60. Nikačević NM, Huesman AEM, Van den Hof PMJ, Stankiewicz Al. Opportunities and challenges for process control in process intensification. Chem Eng Process Process Intensif [Internet]. 2012 Feb [cited $2015 \mathrm{Jul} 1$ 1];52:1-15. Available from:

http://www.sciencedirect.com/science/article/pii/S0255270111002509

61. Kenig E, Jakobsson K, Banik P, Aittamaa J, Górak A, Koskinen M, et al. An integrated tool for synthesis and design of reactive distillation. Chem Eng Sci [Internet]. 1999 May [cited 2015 Jul 1];54(10):1347-52. Available from: http://www.sciencedirect.com/science/article/pii/S0009250999000718

62. Lode F, Houmard M, Migliorini C, Mazzotti M, Morbidelli M. Continuous reactive chromatography. Chem Eng Sci. 2001;56(2):269-91.

63. Pamme N. Continuous flow separations in microfluidic devices. Lab Chip [Internet]. The Royal Society of Chemistry; 2007 Dec 20 [cited 2015 Jun 11];7(12):1644. Available from:

http://pubs.rsc.org/en/content/articlehtml/2007//c/b712784g

64. Lorenz H, Sheehan P, Seidel-Morgenstern A. Coupling of simulated moving bed chromatography and fractional crystallisation for efficient enantioseparation. J Chromatogr A. 2001;908(1-2):201-14.

65. Kaspereit M, Gedicke K, Zahn V, Mahoney AW, Seidel-Morgenstern A. Shortcut method for evaluation and design of a hybrid process for enantioseparations. J Chromatogr A. 2005;1092(1):43-54.

66. Elsner MP, Ziomek G, Seidel-Morgenstern A. Simultaneous preferential crystallization in a coupled batch operation mode. Part II: Experimental study and model refinement. Chem Eng Sci [Internet]. Elsevier; 2011;66(6):1269-84. Available from: http://dx.doi.org/10.1016/j.ces.2010.12.035

67. Ströhlein G, Schulte M, Strube J. Hybrid Processes: Design Method for Optimal Coupling of Chromatography and Crystallization Units. Sep Sci Technol [Internet]. Taylor \& Francis Group; 2003 Jan 9 [cited 2015 Jul 1];38(14):3353-83. Available from: http://www.tandfonline.com/doi/abs/10.1081/SS120023404

68. Howard DW, Lightfoot EN, Hirschfelder JO. The hydrodynamic resolution of optical isomers. AIChE J [Internet]. 1976 Jul [cited $2015 \mathrm{Jul}$ 1];22(4):794-8. Available from:

http://doi.wiley.com/10.1002/aic.690220425

69. Eichhorn R. Enantioseparation in microfluidic channels. Chem Phys [Internet]. Elsevier B.V.; 2010;375(23):568-77. Available from: http://dx.doi.org/10.1016/j.chemphys.2010.06.021

This article is protected by copyright. All rights reserved. 
70. Aristov M, Eichhorn R, Bechinger C. Separation of chiral colloidal particles in a helical flow field. Soft Matter [Internet]. The Royal Society of Chemistry; 2013 Jan 30 [cited 2015 Jul 1];9(8):2525. Available from: http://pubs.rsc.org/en/content/articlehtml/2013/sm/c2sm27441h

71. Hermans TM, Bishop KJM, Stewart PS, Davis SH, Grzybowski BA. Vortex flows impart chirality-specific lift forces. Nat Commun [Internet]. Nature Publishing Group; 2015 Jan 12 [cited 2015 Jul 1];6:5640. Available from: http://www.nature.com/ncomms/2015/150112/ncomms6640/abs/ncomms6640.html

72. Bogunovic L, Fliedner M, Eichhorn R, Wegener S, Regtmeier J, Anselmetti D, et al. Chiral Particle Separation by a Nonchiral Microlattice. Phys Rev Lett [Internet]. 2012 Sep 6 [cited 2015 Jul 1];109(10):100603. Available from: http://link.aps.org/doi/10.1103/PhysRevLett.109.100603

73. Op de Beeck J, De Malsche W, De Moor P, Desmet G. Hydrodynamic chromatography separations in micro- and nanopillar arrays produced using deep-UV lithography. J Sep Sci [Internet]. 2012 Aug 2 [cited 2015 Jul 1];35(15):1877-83. Available from: http://www.ncbi.nlm.nih.gov/pubmed/22865753

74. Op De Beeck J, Callewaert M, Ottevaere H, Gardeniers H, Desmet G, De Malsche W. Suppression of the sidewall effect in pillar array columns with radially elongated pillars. J Chromatogr A [Internet]. 2014 Nov 7 [cited 2015 Jul 1];1367:118-22. Available from: http://www.sciencedirect.com/science/article/pii/S0021967314014861

75. Fonverne A, Demesmay C, Ricoul F, Rouvière E, Dijon J, Vinet F. New carbon nanotubes growth process in a closed microfabricated channel for liquid chromatography application. Sensors Actuators $A$ Phys [Internet]. 2011 Jun [cited 2015 Apr 30];167(2):517-23. Available from: http://www.sciencedirect.com/science/article/pii/S0924424711001142

76. Detobel F, Eghbali H, De Bruyne S, Terryn H, Gardeniers H, Desmet G. Effect of the presence of an ordered micro-pillar array on the formation of silica monoliths. J Chromatogr A [Internet]. 2009 Oct 30 [cited 2015 Jul 1];1216(44):7360-7. Available from: http://www.sciencedirect.com/science/article/pii/S0021967309003720

This article is protected by copyright. All rights reserved. 